\title{
Phylogenetic mapping of bacterial morphology
}

\author{
Janet L. Siefert' $\mathrm{T}$ and George E. Fox ${ }^{2}$ \\ Author for correspondence: George E. Fox. Tel: +1713743 8363. Fax: +1 17137438351. \\ e-mail: fox@uh.edu
}

1 Department of Biology, University of Houston, Houston, TX 77204-5513, USA

2 Department of Biochemical and Biophysical Sciences, University of Houston, Houston, TX 77204-5934, USA

\begin{abstract}
The availability of a meaningful molecular phylogeny for bacteria provides a context for examining the historical significance of various developments in bacterial evolution. Herein, the classical morphological descriptions of selected members of the domain Bacteria are mapped upon the genealogical ancestry deduced from comparison of small-subunit rRNA sequences. For the species examined in this study, a distinct pattern emerges which indicates that the coccus shape has arisen and accumulated independently multiple times in separate lineages and typically survived as a persistent end-state morphology. At least two other morphologies persist but have evolved only once. This study demonstrates that although bacterial morphology is not useful in defining bacterial phylogeny, it is remarkably consistent with that phylogeny once it is known. An examination of the experimental evidence available for morphogenesis as well as microbial fossil evidence corroborates these findings. It is proposed that the accumulation of persistent morphologies is a result of the biophysical properties of peptidoglycan and their genetic control, and that an evolved body-plan strategy based on peptidoglycan may have been a fate-sealing step in the evolution of Bacteria. More generally, this study illustrates that significant evolutionary insights can be obtained by examining biological and biochemical data in the context of a reliable phylogenetic structure.
\end{abstract}

Keywords: bacterial morphology, phylogeny mapping, last common ancestor, peptidoglycan

\section{INTRODUCTION}

Classical morphological descriptions of prokaryotes are simple. Prokaryotes are usually unicellular, small, and typically do not exhibit enough morphological variation to readily define genera or higher phylogenetic groupings as is possible with eukaryotes. Generally, they can be defined as rods, variations of rods, or cocci. More specifically, they can display a range of morphologies within these forms. These include filaments (elongated bacterial cells in which the length exceeds the width, as a sheathed or unsheathed chain of cells), hyphae (branched or unbranched filaments), or in some cases a range of morphologies that involve cellular differentiation/life cycles (e.g. cyanobacteria, actinomycetes). However, members of the domain Bacteria can be identified by their exclusive use of peptidoglycan as the principal constituent for their cell walls. This constituent exhibits two major structural modifications.

†Present address: Department of Statistics, Rice University, Houston, TX, USA.
It is usually claimed to be a monomolecular layer in Gram-negative bacteria (but it may not be; see Labischinski \& Maidhof, 1994). Alternatively, in the Grampositive bacteria, the peptidoglycan forms extensively cross-linked multi-molecular layers. The type of peptidoglycan is thus a reliable but only very broad biochemical/morphological characterization of species in the domain Bacteria. This Gram-positive/Gram-negative dichotomy has been confirmed by a molecular phylogeny based on 16S rRNA sequence information (Fox et al., 1980; Woese et al., 1990, 1985). The molecular phylogeny goes much further, however, by producing reliable relationships throughout the domain Bacteria.

The inherent value of the molecular phylogeny is that it purports to be a natural classification system reflecting actual historical relationships. The early $16 \mathrm{~S}$ rRNA work indicated that some species that had been grouped by morphological criteria were in fact not specifically related. Notable examples included the actinomycetes (Stackebrandt \& Woese, 1981) and the helical bacteria (Woese et al., 1982). Although the Gram reactions, 
endospores, and to an extent spore shape, proved to be meaningful markers, even here there were exceptions. Thus, certain asporogenous, coccoid species are more closely related to members of the genus Bacillus than to their own classically defined bacilli (Stackebrandt, 1988). It is now generally believed that bacterial morphology is largely insignificant in the context of evolutionary history. Is this correct? Herein, we re-examine the issue of whether or not bacterial shape reflects phylogenetic history by mapping classical morphological descriptions from literature surveys upon the genealogical ancestry represented by molecular sequence analysis of smallsubunit rRNA. We are not attempting to resurrect morphology as a phylogenetic tool. Rather, we are seeking to understand, from a phylogenetic standpoint, the evolution of shape in the evolution of Bacteria.

\section{METHODS}

A representative phylogenetic tree based on comparative analysis of small-subunit rRNA of 180 bacterial species was obtained from the Ribosomal Database Project (Maidak et al., 1997). These 180 organisms include all of the major groups of known bacteria for which the small-subunit rRNA has been sequenced (Olsen et al., 1994). A survey of the literature on bacterial cell shape was conducted for each of the 180 organisms. The morphology of each organism was recorded as being rod, coccus, filamentous, spirochaete, helical, or lacking cell walls (mycoplasma). Rod shape was defined as any single cell that is longer than it is wide. Unusual morphology due to nutrient deprivation or environmental conditions was disregarded. Filamentous morphology was recorded only if that morphology was a consistent phenotype not related to growth conditions. The results are depicted in Fig. 1.

Several branches of the representative tree were heterogeneous with respect to distribution of cell shape. Therefore, a detailed investigation of the same type as above was undertaken for one of these heterogeneous branches, the low-G+C Grampositive group. This grouping included 260 species from the families Lactobacillaceae, Streptococcaceae and Bacillaceae; the results are seen in Fig. 2.

In a study of this type, it is necessary to address the complication presented by lineages that exhibit high degrees of morphological diversity through life cycles and/or primitive multicellularity. Two of the most notable of these are the high$\mathrm{G}+\mathrm{C}$ Gram-positive lineage and the cyanobacterial lineage. However, our understanding of the genetic control of bacterial lifestyles as described in the high-G $+C$ Gram-positives, and available in-depth phylogenetic analyses, are limiting. The phylogenetic data available for the cyanobacteria were investigated with the caveat that they are among the only prokaryotes with a substantiated microfossil record (Schopf, 1994). Their range of morphology includes both true and false branching, with the individual cell shape often being influenced by the arrangement of cells in aggregates. These organisms have apparently undergone an exceptionally low rate of morphological change over hundreds or thousands of millions of years (hypobradytely) such that in many instances, tentative identification of forms seen in the fossil record has been made to the genus/species level (Schopf, 1994). The cyanobacteria were investigated in the same manner as above, with the exception that the phylogenetic tree was obtained from Giovannoni et al. (1988) and includes 31 species.

\section{RESULTS}

Fig. 1 is the phylogenetic tree from the initial survey including 180 species of bacteria. This tree estimates the number of changes between sequences and displays which bacteria are most closely related to each other. The Archaeal branch roots the tree and the deepest branches represent the extant relatives of the oldest, most ancient bacteria (Olsen et al., 1994; Woese, 1987; Achenbach-Richter et al., 1988). The branches are coded to indicate the results of the mapping. As shown, the deepest branches of the tree contain exclusively rodshaped, often filamentous organisms. Filamentous lineages are also found in at least six of the major branches. Due to the ability of some micro-organisms to exhibit filamentation under certain environmental conditions, strict assignments for this phenotype were difficult. However, some lineages (e.g. cyanobacteria and Planctomyces) appear to contain clusters that are persistent for those morphologies. Helical (spirillum) species were found in four lineages of Gram-negative bacteria (delta and epsilon, alpha, gamma, and beta). The spirochaetes and the mycoplasmas have unique morphologies and are represented by monophyletic lines of descent in the Bacterial 16S rRNA tree. The remaining lineages are classified as homogeneous for rod-shape (Fibrobacter and Cytophaga/Flexibacter/Bacteroides), homogeneous for cocci and/or filamentous bacteria (Deinococcus), or heterogeneous, e.g. exhibiting rod and coccus morphology within the same lineage.

An unexpected finding was the striking pattern of rod versus coccus morphology with respect to the homogeneity/heterogeneity within clusters. Throughout the tree, clusters with predominantly rod morphology were frequently heterogeneous, with species exhibiting both rod and coccus morphology present. However, once a particular lineage exhibited coccus morphology, the cluster or clusters resulting from that lineage invariably became homogeneous for the coccus morphology. This suggests that once formed, the coccus morphology may be persistent throughout subsequent bacterial evolution. This frequent degeneration to the coccus morphology has been noted previously (Stackebrandt \& Woese, 1981 ), but the full scope of its significance was not clear at that time.

Fig. 2 shows the result of a more detailed examination of a portion of the low-G $+C$ Gram-positive lineage that exhibited extensive heterogeneity from Fig. 1. These organisms were represented by a tree containing 260 species from the families Lactobacillaceae, Streptococcaceae and Bacillaceae. In this lineage the repeated and persistent evolution of the coccus phenotype was quite robust. Throughout the lineage, repeated discoveries of coccus-shaped organisms are never seen to revert in later lineages to rod-shaped morphology. This suggests that while the coccus morphology has evolved many times in prokaryotic history, it represents a persistent end-state for bacterial cell shape. The alternative hypothesis, that the ancestor was a coccus, is far less parsimonious.

Using the small-subunit rRNA tree of Giovannoni et al. 


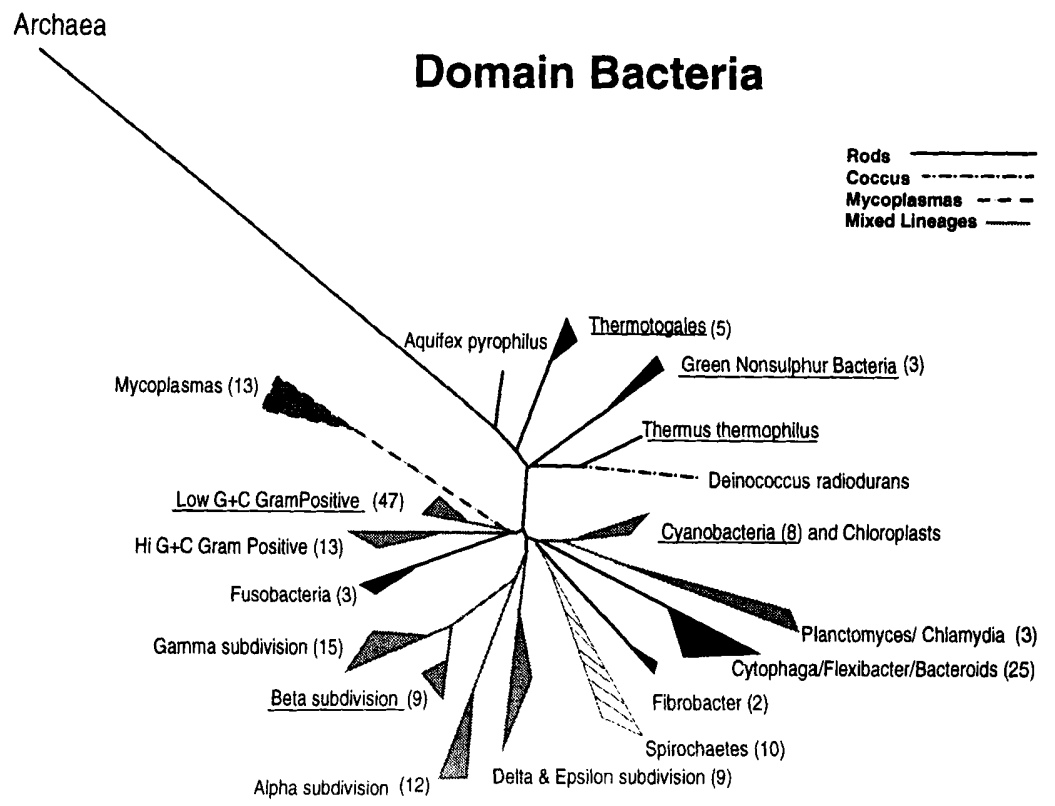

Fig. 1. Phylogenetic mapping of bacterial morphologies on the Ribosomal Database Project (RDP) tree of the domain Bacteria based on 16S rRNA gene sequences. Individual branches are coded as indicated on the figure. Those lineages that are underlined include filamentous forms. For simplicity the branches within each major group were collapsed and the breadth of divergence displayed by boundaries of the most distant members of each lineage. The number of species investigated for that branch is shown in parentheses. Morphological description and/or information for references to original papers was taken from two major sources (Sneath, 1984; Balows et al., 1992).The RDP Database was accessed through the WorldWide Web (http://www.cme.msu.edu).

\section{Order Bacillales}

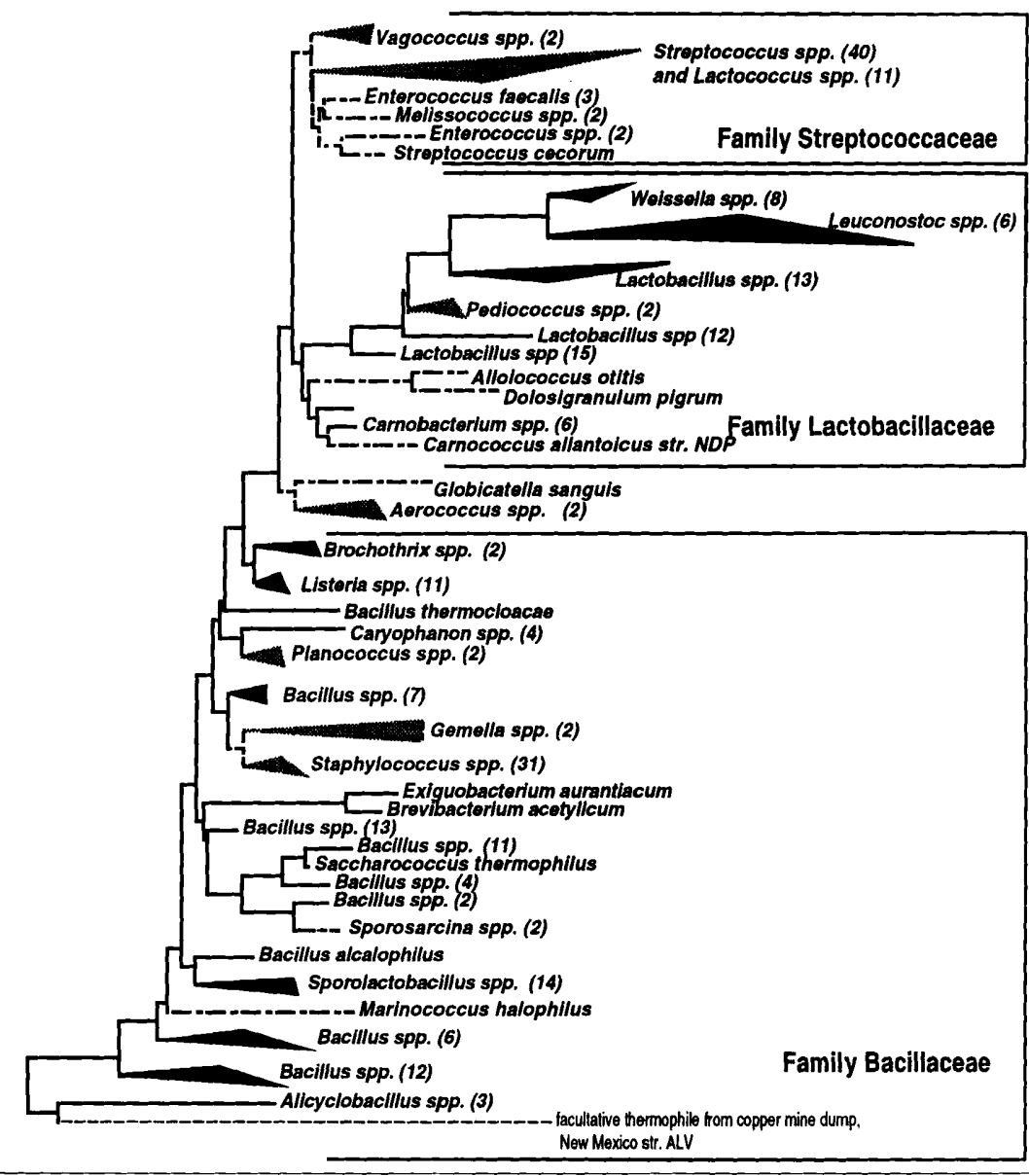

Fig. 2. Comprehensive RDP tree based on the 165 rRNA gene sequences of members of the Order Bacillales mapped with classical morphologies. The Order Bacillales is one branch of the low- $G+C$ lineage from Fig. 1. Lineages are coded: dashed/dotted line with grey shading designates coccus morphology; solid black indicates rod morphology. Species designations were replaced with the name of the genera of the cluster and the numbers of species evaluated for each cluster are in parentheses. Morphological description source information was obtained as in Fig. 1.

(1988), 31 species of cyanobacteria were evaluated as previously described. No discernible morphological pattern for the species of cyanobacteria was observed, except for the clustering of filamentous heterocystous cyanobacterial species (Giovannoni et al., 1988). Heterocysts appear only recently in the fossil record and 
must have diverged sometime after the split of the cyanobacteria from the Gram-positive and Gram-negative lineages (Feng et al., 1997). This is fully consistent with their restriction to a single recent cluster in the cyanobacterial tree. However, it is interesting to note that a number of the cyanobacteria appear to have acquired persistent end-state morphologies as early as 3.5 billion years ago (Giovannoni et al., 1988). This persistence of coccoid and filamentous forms in the cyanobacterial lineage has facilitated interpretation of their fossil record. In light of the present study, it is an impressive illustration of the difficulty of recovering the rod morphology once it is lost.

\section{DISCUSSION}

The correlation between cell shape and the phylogeny represented by the trees clearly demonstrates that there is evolutionary significance to bacterial morphology. Interestingly, this was initially obscured by the fact that some morphologies appear to evolve repeatedly (cocci, filamentous forms, and helical shape) after which they persist, while others represent singular but equally persistent events (spirochaetes and mycoplasmas). Once one understands that certain morphologies, e.g. the coccus, have been repeatedly and independently discovered, the congruence between cell shape and the molecular data becomes striking. The phylogenetic mapping methodology employed here allowed tracking of evolutionary trends that were previously impossible to detect. It is reasonable to suppose that a similar analysis can be applied to a large variety of biochemical characters, e.g. distributions of genes, gene orders, alternative pathways.

A complicating factor encountered in this study involves morphological descriptions of species that exhibit multiple phenotypes, such as Listeria, where older cultures can exhibit a coccus phenotype rather than the "wildtype' rod-shape. Instances of this occurring appear to be directly related to environmental conditions that alter the balance of the enzyme system in such a way that changes in the timing for cell division versus elongation compensate for the unfavourable conditions. These are regulatory events that are most likely moderated by substrate availability, and do not represent changes in genotype that would result in inheritable phenotypes of a particular bacterium. A further example involves filamentous morphologies that depend on the availability of ppGpp for expression of the fts $Z$ gene (Lutkenahus \& Mukherjee, 1996), which temporarily modulates cellular division. There are examples of a more stable expression of filamentous forms (independent of environmental stress) as seen in cyanobacterial species and the early branching organisms Aquifex and Thermotogales (Fig. 1) which are indications of a persistent phenotype.

The existence of life cycles in bacteria, especially the high-G + C Gram-positives (Balows et al., 1992), represents an important development in the evolution of Bacteria. These genera have evolved cellular diffe- rentiation and segregation that allow differential developmental morphology and multicellularity. Clues to the ability of bacteria to evolve into multicellular entities with life cycles may assist our understanding of the eukaryotic domain's successful exploitation of cellular complexity. In this context, we consider the high-G + C Gram-positive organisms as being subject to the same physical properties that dictate cellular morphology as in the classic bacteria, but with highly sophisticated genetic systems that allow for intricate morphological variation beyond what is normally seen. It should be noted that within the high-G + C Gram-positive lineage, the majority of species exhibit intricate life cycles or diverse morphologies as in Rothia dentocariosa (Gerencser $\&$ Bowden, 1986). Hence, it can be argued that the last common ancestor to this lineage had already evolved the complex genetic system required to support a developmental cycle, and that this represents a novel evolutionary strategy in place quite early in the domain Bacteria. In essence, the life-cycle strategy is itself another example of a uniquely evolved end-state morphology

Instances of genera that are recalcitrant to taxonomic placement are rare, but do exist. An example is the genus Pasteuria (Ebert et al., 1996). An attempt to reconcile Pasteuria morphology, development and biochemistry from a phylogenetic perspective (Ebert et al., 1996; Stackebrandt et al., 1984) led to the hypothesis that this genus is related to the genus Alicyclobacillus. The low bootstrap values $(28 \%)$ and lack of obvious biochemical similarity make this affinity a tenuous conjecture. Genera of this type have not been included in the present study, which requires a reliable phylogeny.

Bacterial shape is a result of the biochemical and biophysical properties of peptidoglycan within the cell walls (Schleifer \& Kandler, 1972). An understanding of the role and regulation of peptidoglycan synthesis is necessary for interpretation of the results of this study as well as an interpretation of what if any of the morphological phenotypes are phylogenetically important. Early taxonomic work based on peptidoglycan variation within the Bacteria correlates well with the phylogeny provided by rRNA sequence analysis. Gram-negative bacteria and cyanobacteria use, almost exclusively, a direct cross-link with the diamino acid at position 3 of the peptide subunit. In contrast, Gram-positive bacteria utilize this type of linkage as well as a wide variety of others that involve inter-peptide bridges and novel amino acids such as lysine and ornithine.

Peptidoglycan and cell wall synthesis involves a complex and intricate coordination of at least two morphogenic systems (Park, 1996; Begg et al., 1986). A great deal is understood concerning the major enzymes of these systems (Begg et al., 1990; Spratt et al., 1980; Tamaki et al., 1980; Lutkenhaus \& Mukherjee, 1996; Costa \& Anton, 1993), but little is understood concerning the actual control of cell elongation and cell division, which will ultimately determine cell shape. Experimental evidence with Escherichia coli and Salmonella indicates 
that small fluctuations in expression can lead to a variety of phenotypes, e.g. unrecoverable coccus morphology, antibiotic resistance, filamentation, and/or improper cell division/non-viable mutants (Begg et al., 1986; Costa \& Anton, 1993; Pollack \& Neuhaus, 1994). The survival or nonsurvival of genetic mutants depends on the competing system that is altered and the type of mutation. The enzyme system controlling peptidoglycan synthesis, morphogenesis and cell division should be viewed as a system that can be altered at two levels: one which can compensate for environmental stresses and is temporal in nature, affecting only the phenotype; and one which represents actual genetic mutations, changing the genotype, and which may favour persistent phenotypes - most notably from this study, the coccus morphology.

Therefore, current biochemical evidence points to the coccus morphology as a degenerative form of the rod. In addition, filamentous forms of morphology that are not environmentally dependent are persistent phenotypes in certain lineages. Both of these morphologies are widespread throughout the domain Bacteria. However, the spirillum morphology represents an exclusively Gramnegative invention. This is most likely due to the single layer of peptidoglycan in the cell walls of spirilla, which would confer less rigidity. The spirillum may represent common end-state morphology as well, but the data are too limited to adequately test this.

We propose that the properties of peptidoglycan synthesis, and the intimate association of cell elongation and cell division, lead to these persistent or end-state morphologies in the Bacteria. Their persistence results from the inheritable changes in the genes that control peptidoglycan synthesis and morphogenesis and in some cases may provide an adaptive advantage, such as the functional multicellularity seen in the heterocystous cyanobacteria (Giovannoni et al., 1988), the developmental cycles of the high-G $+C$ Gram-positives (Balows et al., 1992) or the antibiotic resistance exhibited in Salmonella, which compensates for losses in certain cellshape-determining genes (Costa \& Anton, 1993). In the end, however, it would appear the early choice of the peptidoglycan sacculus greatly restricted the ability of the Bacteria to evolve complex structures.

It also seems likely that the last common ancestor of the domain Bacteria was rod-shaped. This is based on the presence of exclusively rod-shaped organisms in the deepest lineages of the phylogenetic tree and the role of peptidoglycan as the solely evolved body plan strategy for the Bacteria. The evolutionary separation and divergence of eukaryotes and prokaryotes is evidenced by two separate strategies of cell evolution. The prokaryotes develop a cell wall to combat osmotic pressure, whereas the eukaryotes use cytoskeleton/mechanoprotein. Within the two prokaryotic lineages, the Bacteria and the Archaea, different cell wall constituents have evolved (Schleifer \& Kandler, 1972). No archaeon has a true peptidoglycan sacculus and in fact several different cell wall polymers are found; hence the presence of peptidoglycan distinguishes the Bacteria from the Archaea (Kandler \& Hippe, 1977). This suggests that exploration of a possible choice of a sacculus was ongoing before the Archaea/Bacteria divergence. By the time of the last common ancestor to the Bacteria, peptidoglycan was well established as the major constituent of the cell wall. Much as the establishment of animal body plans by the close of the Cambrian explosion would subsequently involve only basic modification of current architecture (Valentine $e t$ al., 1996), evolution in the domain Bacteria was, from this point forward, limited by the biochemical and biophysical constraints of peptidoglycan. Interestingly, evolution managed to circumvent the constraints imposed by peptidoglycan to evolve two novel morphologies. The mycoplasmas (intracellular parasites without cell walls) and the spirochaetes (cells that contain periplasmic flagella that wind around a helical protoplasmic cylinder) are distinct morphologies which the $16 S$ rRNA tree suggests were invented once in the history of the Bacteria. These are probably also persistent end-state morphologies.

\section{ACKNOWLEDGEMENTS}

We thank Dr Stanley Miller for helpful discussions. This work was supported in part by grants from NASA (NAG5-4004) and the Institute of Space Systems Operations at the University of Houston to G.E.F.

\section{REFERENCES}

Achenbach-Richter, L., Gupta, R., Zillig, W. \& Woese, C. R. (1988). Rooting the archaebacterial tree: the pivotal role of Thermococcus celer in archaebacteria evolution. Syst Appl Microbiol 9, 34-39.

Balows, A., Trüper, G., Dworkin, M., Harder, W. \& Schleifer, K.-H. (1992). The Prokaryotes, 2nd edn. New York: Springer.

Begg, K. J., Spratt, B. G. \& Donachie, W. D. (1986). Interaction between membrane proteins PBP3 and RodA is required for normal cell shape and division in Escherichia coli. J Bacteriol 167, 1004-1008.

Begg, K. J., Taskasuga, A., Edwards, D. H., Dear, S. J., Spratt, B. G., Adachi, H., Ohta, T., Matsuzawa, H. \& Donachie, W. D. (1990). The balance between different peptidoglycan precursors determines whether Escherichia coli cells will elongate or divide. $J$ Bacteriol 172, 6697-6703.

Costa, C. S. \& Anton, D. N. (1993). Round-cell mutants of Salmonella typhimurium produced by transposition mutagenesis: lethality of $\operatorname{rodA}$ and mre mutations. Mol Gen Genet 236, 387-394.

Ebert, D., Rainey, P., Embley, T. M. \& Scholz, D. (1996). Development, life cycle, ultrastructure and phylogenetic position of Pasteuria ramosa Metchnikoff 1888: rediscovery of an obligate endoparasite of Daphnia magna Straus. Philos Trans $R$ Soc Lond B 351, 1689-1701.

Feng, D. F., Cho, G. \& Doolittle, R. F. (1997). Determining divergence times with a protein clock: update and re-evaluation. Proc Natl Acad Sci USA 94, 13028-13033.

Fox, G. E., Stackebrandt, E., Hespell, R. B. \& 16 other authors (1980). The phylogeny of prokaryotes. Science 209, 457-463.

Gerencser, M. A. \& Bowden, G. H. (1986). Genus Rothia. In Bergey's Manual of Systematic Bacteriology, vol. 2, pp. 13421344. Edited by P. H. A. Sneath. Baltimore: Williams $\&$ Wilkins. 
Giovannoni, S. J., Turner, S., Olsen, G. J., Barnes, S., Lane, D. J. \& Pace, N. R. (1988). Evolutionary relationships among cyanobacteria and green chloroplasts. J Bacteriol 170, 3584-3592.

Kandler, O. \& Hippe, H. (1977). Lack of peptidoglycan in the cell walls of Methanosarcina barkeri. Arch Microbiol 113, 57-60.

Labischinski, H. \& Maidhof, H. (1994). Bacterial peptidoglycan: overview and evolving concepts. In Bacterial Cell Wall, pp. 23-38. Edited by J.-M. Ghuysen \& R. Hakenbeck. Amsterdam: Elsevier.

Lutkenhaus, J. \& Mukherjee, A. (1996). Cell division. In Escherichia coli and Salmonella typhimurium, pp. 1615-1626. Edited by F.C. Neidhardt and others. Washington, DC: American Society for Microbiology.

Maidak, B. L., Olsen, G. J., Larsen, N., Overbeek, R., McCaughey, M. J. \& Woese, C. R. (1997). The RDP (ribosomal database project). Nucleic Acids Res 25, 109-111.

Olsen, G. J, Woese, C. R. \& Overbeek, R. (1994). The winds of (evolutionary) change: breathing new life into microbiology. J Bacteriol 176, 1-6.

Park, J. T. (1996). The murein sacculus. In Escherichia coli and Salmonella typhimurium, pp. 48-57. Edited by F. C. Neidhardt and others. Washington, DC: American Society for Microbiology.

Pollack, J. H. \& Neuhaus, F. C. (1994). Changes in wall teichoic acid during the rod-sphere transition of Bacillus subtilis 168. $J$ Bacteriol 176, 7252-7259.

Schleifer, J. H. \& Kandler, O. (1972). Peptidoglycan types of bacterial cell walls and their taxonomic implications. Bacteriol Rev 36, 407-477.

Schopf, J. W. (1994). Disparate rates, differing fates: tempo and mode of evolution changed from the Precambrian to the Phanerozoic. Proc Natl Acad Sci USA 91, 6735-6742.

Sneath, P. H. A. (1984). Endospore-forming Gram-positive rods and cocci. In Bergey's Manual of Systematic Bacteriology, vol. 2, pp. 1104-1207. Edited by N. R. Kreig \& J. G. Holt. Baltimore: Williams \& Wilkins
Spratt, B. G., Boyd, A. \& Stoker, N. (1980). Defective and plaqueforming lambda transducing bacteriophage carrying penicillinbinding protein-cell shape genes: genetic and physical mapping and identification of gene products from the lip-dacA-rodA-pdpleuS region of the Escherichia coli chromosome. J Bacteriol 143, 569-581.

Stackebrandt, E. (1988). Phylogenetic relationships vs. phenotypic diversity: how to achieve a phylogenetic classification system of the eubacteria. Can J Microbiol 34, 552-556.

Stackebrant, E. \& Woese, C. R. (1981). Towards a phylogeny of the Actinomycetes and related organisms. Curr Microbiol 5, 197-202.

Stackebrandt, E., Ludwig, W., Schubert, W., Klink, F., Schlesner, H., Roggentin, T. \& Hirsch, P. (1984). Molecular genetic evidence for early evolutionary origin of budding peptidoglycan-less eubacteria. Nature 307, 735-737.

Tamaki, S., Matsuzawa, H. \& Matsuhashi, M. (1980). Cluster of $m r d A$ and $m r d B$ genes responsible for the rod shape and mecillinam sensitivity of Escherichia coli. J Bacteriol 141, 52-57.

Valentine, J. W., Erwin, D. H. \& Jablonski, D. (1996). Developmental evolution of metazoan body plans: the fossil evidence. Dev Biol 173, 373-381.

Woese, C. R. (1987). Bacterial evolution. Microbiol Rev 51, 221-271.

Woese, C. R., Blanz, P., Hespell, R. B. \& Hahn, C. M. (1982). Phylogenetic relationships among various helical bacteria. Curr Microbiol 7, 119-124.

Woese, C. R., Stackebrandt, E., Macke, T. J. \& Fox, G. E. (1985). A phylogenetic definition of the major eubacterial taxa. Syst Appl Microbiol 6, 143-151.

Woese, C. R., Kandler, O. \& Wheelis, M. L. (1990). Towards a natural system of organisms: proposal for the domains Archaea, Bacteria, and Eucarya. Proc Natl Acad Sci USA 87, 4576-4579.

Received 2 March 1998; revised 8 June 1998; accepted 17 June 1998. 